\title{
Investigation of vibration parameters for needle insertion force reduction
}

Dennis Rehling, Institute of Medical Device Technology, University of Stuttgart, Stuttgart, Germany, st144547@stud.uni-stuttgart.de

Jan Liu, Institute of Medical Device Technology, University of Stuttgart, Stuttgart, Germany, jan.liu@imt.uni-stuttgart.de

Frank Schiele, Institute for Design and Manufactoring in Precision Engineering, University of Stuttgart, Stuttgart, Germany, frank.schiele@ikff.uni-stuttgart.de

Kent W. Stewart, Institute of Medical Device Technology, University of Stuttgart, Stuttgart, Germany, kent.stewart@imt.uni-stuttgart.de

Peter P. Pott, Institute of Medical Device Technology, University of Stuttgart, Stuttgart, Germany, peter.pott@imt.uni-stuttgart.de

\section{Introduction}

Drawing of blood for diagnostic testing is the most common invasive procedure performed in healthcare. Despite the frequency and importance of venepuncture, complications such as severe pain and formation of hematoma caused by positioning errors occur frequently. It has been shown that pain experience and needle positioning can be improved by a reduction of insertion force which can be achieved by vibrating the needle axially. This paper investigates different combinations of vibration frequencies and vibration amplitudes to minimize insertion force.

\section{Methods}

A $21 \mathrm{G}$ hypodermic needle was vibrated by attaching it to a voice coil with an adapter. A laser vibrometer was used to adjust the excitation voltage for specific free vibration amplitudes $(20,100$, and $500 \mu \mathrm{m})$ and frequencies $(10,100$, and $200 \mathrm{~Hz})$. A $125 \mu \mathrm{m}$ thin PET skin simulant was attached to a load cell and driven towards the vibrating needle by a linear motor. The resulting force curves for different combinations of amplitudes and frequencies were recorded by a measuring amplifier and analysed in terms of force reduction.

\section{Results}

Force curves show characteristic points and phases during puncture. An initial puncture force as well as the force for widening the crack by the first bevel of the hypodermic needle and the cutting force induced by the second bevel can be seen. Adding an axial vibration to the linear insertion movement increases the momentary force at the tip as the velocities of vibration and linear insertion movement add up. As a result, the insertion force needed to puncture a skin is always lower when vibrating the needle axially.

\section{Conclusion}

Reduction of insertion force was achieved by vibrating a needle axially. In further experiments, more combinations of frequencies and amplitudes will be tested as well as the force progression in complete tissue phantoms. 


\section{Workflow and Human-Centered Risk Analysis for Novel Mechatronic Rescue Aids}

Drobinsky Sergey, Chair of Medical Engineering at Helmholtz-Institute for Biomedical Engineering, RWTH Aachen University, Aachen, Germany,drobinsky@hia.rwth-aachen.de

Verjans Mark, Chair of Medical Engineering at Helmholtz-Institute for Biomedical Engineering, RWTH Aachen University, Aachen, Germany, verjans@hia.rwth-aachen.de

Schleer Philipp, Chair of Medical Engineering at Helmholtz-Institute for Biomedical Engineering, RWTH Aachen University, Aachen, Germany, schleer@hia.rwth-aachen.de

Kolk Benedikt, Chair of Medical Engineering at Helmholtz-Institute for Biomedical Engineering, RWTH Aachen University, Aachen, Germany, benedikt.kolk@rwth-aachen.de

Bensiek Henrike, Chair of Medical Engineering at Helmholtz-Institute for Biomedical Engineering, RWTH Aachen University, Aachen, Germany, henrikejohanna.bensiek@rwth-aachen.de

Radermacher Klaus: Chair of Medical Engineering at Helmholtz-Institute for Biomedical Engineering, RWTH Aachen University, Aachen, Germany, radermacher@hia.rwth-aachen.de

Janß Armin: Chair of Medical Engineering at Helmholtz-Institute for Biomedical Engineering, RWTH Aachen University, Aachen, Germany,janss@hia.rwth-aachen.de

\section{Introduction}

Paramedics face rising numbers of deployments every year. As obstacles like stairs occur often, paramedics must frequently manually carry patients and are thereby exposed to loads multitudes higher than recommended. This creates the need for patient transport aids (PTA), which can physically support paramedics in a wide variety of transport situations, without slowing down the transport.

\section{Methods (14 pt bold)}

In this paper a workflow analysis for transport missions in an urban context and basic tasks for PTAs are presented. Subsequently, the high-level task modelling and human-centered risk analysis according to the HiFEM method are presented for the use case of a patient transport over stairs with a passive PTA, like a rescue chair, and an active PTA like the novel SEBARES prototype.

\section{Results (14 pt bold)}

The analysis shows that conventional PTA's have a simple linear use process, however, impose excessive physical workloads, which cause risks like the paramedic or the PTA falling down the stairs. Contrary, active PTA's reduce physical workloads, however, introduce additional concurrent steps, like identifying and correcting misalignments, which create further risks.

\section{Conclusion (14 pt bold)}

In order to mitigate risks with active, stair climbing PTAs either new kinematic designs or intelligent assistance functions, like automatic stair detection, are necessary. 


\section{Additive Manufacturing of Medical Devices facing Regulatory Requirements}

Marian Gransow, VIVE-MedTech GmbH, Cottbus, Germany, marian.gransow@vive-medtech.com Susanne Kromnik, Institute of Biomedical Engineering, TU Dresden, Dresden, Germany, Susanne.kromnik@tu-dresden.de

Andreas Strauß, VIVE-MedTech GmbH, Cottbus, Germany, andreas.strauss@vive-medtech.com

Christine Thiele, Institute of Biomedical Engineering, TU Dresden, Dresden, Germany, Christine.thiele@tudresden.de

\section{Introduction}

Additive manufacturing using Fused Filament Fabrication (FFF) is a promising technique for rapid functional prototyping and small batch production of medical devices. However, functional prototyping and production in the field of medical devices are restricted by a variety of national regulatory requirements for different markets. Many additive manufactured devices remain expensive and not usable for series production.

\section{Methods}

As founders and partners of a start-up utilizing FFF of medical devices like catheters, oxygenators, surgical instruments and others, we analysed actual and upcoming regulatory requirements for medical device manufacturers. Process specific requirements for FFF were mapped into requirements for a Quality Management System (QMS) and products. Based on this framework we developed a unique technical solution of FFF in a clean room environment.

\section{Results}

From the perspective of a medical device manufacturer we implemented a completed QMS according to Regulation 2017/745, EN ISO 13485:2016 and 21 CFR Parts 11 and 820. Herein product development and production, especially design, risk management, ensuring biocompatibility including sterility of materials and contacting process elements as well as supplier management and quality assurance of products are performed. First products are available as prototypes for clinical evaluation.

\section{Conclusion}

FFF and other additive manufacturing techniques can massively extend possibilities of user and application individualized medical devices.

However, medical devices, regardless of their production are regulated. We developed a strategy for cost effective generic design, documentation and production of FFF-produced medical devices. Further this strategy was implemented into a start-up for additive manufactured medical devices.

In the result cost effective FFF of medical devices for special applications with smaller batches are possible. New possibilities for individualized and specialized medical devices are established. 


\title{
Verification of Pressure Tightness of 3D-printed Cannulas for ex vivo Organ Perfusion
}

\author{
Susanne Kromnik, Institute of Biomedical Engineering, TU Dresden, Dresden, Germany, Su- \\ sanne.Kromnik@tu-dresden.de \\ Philipp Talhofer, Institute of Biomedical Engineering, TU Dresden, Dresden, Germany, Philipp.Talhofer@tu- \\ dresden.de \\ Marian Gransow, VIVE-MedTech GmbH, Cottbus, Marian.Gransow@vive-medtech.com \\ Christine Thiele, Institute of Biomedical Engineering, TU Dresden, Dresden, Germany, Christine.Thiele@tu- \\ dresden.de \\ Andreas Strauß, VIVE-MedTech GmbH, Cottbus, Andreas.Strauss@vive-medtech.com \\ Hagen Malberg, Institute of Biomedical Engineering, TU Dresden, Dresden, Germany, Hagen.Malberg@tu- \\ dresden.de
}

\section{Introduction}

Currently, 3D-printing plays an increasing role in manufacturing. Products are customized, fast to manufacture and cost-effective for small batches. For this reason, we developed cannulas from Fused Filament Fabrication (FFF), which are designed to deliver blood-based solutions to an ex vivo perfused organ. The pressure tightness of arterial and venous cannulas is necessary for the safety of organ perfusion. We present a setup and results of pressure tightness testing according to EN ISO 10555-1.

\section{Methods}

A normative specified pressure of at least $300 \mathrm{kPa}$ for $30 \mathrm{~s}$ shall be applied to the cannulas for the leakage test. Therefore, a measurement setting consisting of a pump, a check valve, pressure sensors and a piping system was designed. The pressure was adjusted by a three-point controller. User interface was implemented utilizing an Arduino with LCD touch display. Cannulas were specially developed for FFF from biocompatible PETG material. The used clean room 3d printer was developed by VIVE-MedTech for biocompatible products.

\section{Results}

The test setting was able to generate and measure a pressure of approximately $400 \mathrm{kPa}$. In initial investigations with $0,45 \mathrm{~mm}$ thin-walled cannulas a test pressure of above $300 \mathrm{kPa}$ could be maintained for $30 \mathrm{~s}$. No leakage of liquid could be detected.

\section{Conclusion}

Our measurement setup allows the investigation of pressure tightness of cannulas and catheters according to EN ISO 10555-1. The required pressure tightness of the additive manufactured cannulas could be proven. Cannulas manufactured using FFF are therefore qualified for fluid transport in ex vivo circuits under the aspect of pressure tightness. Further investigations, such as the evaluation of the mechanical biocompatibility of printed cannulas, will follow. 


\section{On the fractal classification of subviral particle motion in fluorescence mi- croscopy sequences of Ebola-infected live-cells}

Andreas Rausch, Institut für Biomedizinische Technik (IBMT), FB Life Science Engineering (LSE), Technische Hochschule Mittelhessen (THM) - University of Applied Sciences, Gießen, Germany, andreas.rausch@lse.thm.de Thomas Schanze, Institut für Biomedizinische Technik (IBMT), FB Life Science Engineering (LSE), Technische Hochschule Mittelhessen (THM) - University of Applied Sciences , Gießen, Germany, thomas.schanze@1se.thm.de

\section{Introduction}

An accurate knowledge of the movement characteristics of subviral Ebola particles is essential for the current research for medicines. An fractal dimension-based algorithm to automatically classify subviral particle motion in image sequences of infected live-cells is presented.

\section{Methods}

Fractal dimension estimation is used as a measure for the complexness of structures or - in this case - tracks. An resolution independent method to estimate a track's fractal dimension is applied to testing data: $F D=\log (n) /[\log (n)+\log (d / L)]$, with $n$, the number of track segments $(N-1) ; d$, the displacement between the two farthest measurements and $L$, the total track length. The method's accuracy was tested on a simulated Koch curve-shaped track. Furthermore it was applied to all subvrial particle tracks of a real infected cell.

\section{Results}

The fractal dimension of the simulated Koch curve-shaped track estimated with the proposed method is 1.263. Comparing the estimated fractal dimension of the Koch curve-like track to the literature (1.262) indicates a high accuracy of the proposed method. Using the results of the fractal analysis a visualization of the motion distribution within the real infected cell is created. This allows spatio-temporal fractal dimension mapping of subviral particle tracks.

\section{Conclusion}

The resolution-independence of the approach makes it more reliable than the recently tested box count-based method. The visualization of fractal dimensions enables a fast and easy presentation of motion characteristics for pharmaceutical researchers. 